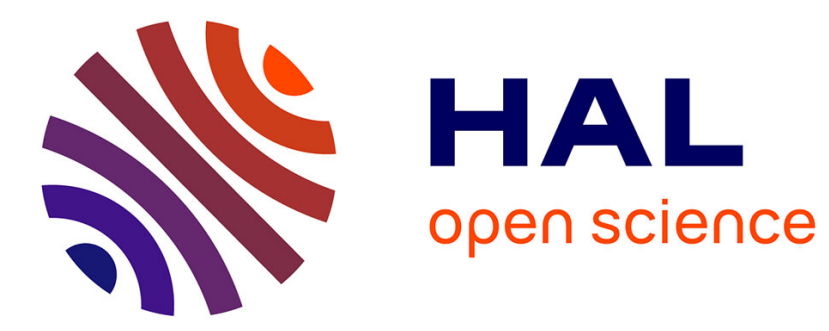

\title{
Effect of rf on subharmonic gap structure in superconducting junctions
}

O. Hoffmann Soerensen, B. Kofoed, N.F. Pedersen, S. Shapiro

\section{To cite this version:}

O. Hoffmann Soerensen, B. Kofoed, N.F. Pedersen, S. Shapiro. Effect of rf on subharmonic gap structure in superconducting junctions. Revue de Physique Appliquée, 1974, 9 (1), pp.153-155. 10.1051/rphysap:0197400901015300 . jpa-00243725

\section{HAL Id: jpa-00243725 https://hal.science/jpa-00243725}

Submitted on 1 Jan 1974

HAL is a multi-disciplinary open access archive for the deposit and dissemination of scientific research documents, whether they are published or not. The documents may come from teaching and research institutions in France or abroad, or from public or private research centers.
L'archive ouverte pluridisciplinaire HAL, est destinée au dépôt et à la diffusion de documents scientifiques de niveau recherche, publiés ou non, émanant des établissements d'enseignement et de recherche français ou étrangers, des laboratoires publics ou privés. 


\title{
EFFECT OF rf ON SUBHARMONIC GAP STRUCTURE IN SUPERCONDUCTING JUNCTIONS
}

\author{
O. HOFFMANN SOERENSEN, B. KOFOED, \\ N. F. PEDERSEN and S. SHAPIRO $\left({ }^{*}\right)$ \\ Physics Laboratory I, The Technical University of Denmark, \\ DK-2800 Lyngby, Denmark
}

\begin{abstract}
Résumé. - On a mesuré l'influence de la puissance rf sur la structure de la caractéristique courant-tension pour les points donnés par la relation $V=(2 \Delta \pm n h v) / m e(2 \Delta$ est la bande interdite du supraconducteur, $v$ la fréquence $\mathrm{rf}, m=1,2,3, \ldots$ et $n=0,1,2, \ldots$ ). Les résultats obtenus sont très semblables à ceux correspondants à un effet tunnel assisté; c'est-à-dire que la hauteur des marches suit une loi de la forme $J_{n}^{2}\left(m \mathrm{e} V_{\mathrm{rr}} / h v\right)$ expression dans laquelle $J_{n}(x)$ est une jonction de Bessel et $V_{\mathrm{re}}$ la tension rf appliquée à la jonction.

Abstract. - The rf-power dependence of the structure in the current-voltage characteristic located at the voltage $V=(2 \Delta \pm n h v) / m$ e has been measured and found very similar to the familiar microwave-assisted tunneling $(m=1) .2 \Delta$ is the superconducting energy gap, $v$ the applied frequency, $m=1,2,3, \ldots$ and $n=0,1,2, \ldots$ That is, the variation with power of all these structures is found consistent with the expression $J_{n}^{2}\left(m \mathrm{e} V_{\mathrm{rf}} / h v\right)$ where $J_{n}(x)$ is the ordinary Bessel function and $V_{\mathrm{rf}}$ the microwave voltage across the junction.
\end{abstract}

In addition to the structure in the current-voltage $(I-V)$ characteristic of junctions between identical superconductors at the voltage $V=2 \Delta / \mathrm{e}(2 \Delta$ is the energy gap), structure is commonly observed also at submultiples of this voltage, i. e., at $V=2 \Delta / m$ e where $m$ is an integer. The origin of this so-called « subharmonic " gap structure (SGS) is not yet resolved although many attempts to do so have been made in the past decade. See, e. g., the paper of Rowell and Feldman [1] which discusses the phenomenon at length and contains an extensive bibliography.

Mainly two explanations of the structure have been suggested, namely multiparticle tunneling [2] and nonlinear self-coupling of the Josephson radiation [3]. Since the latter explanation invokes the ac-Josephson effect directly whereas the former does not, one might hope that a distinction between the two explanations can be established experimentally by means of an externaliy applied microwave radiation (rf). Giaever and Zeller [4] found, in fact, that the SGS was strongly dependent on the presence of rf-fields, and Longacre and Shapiro [5] suggested that the rf-response of the SGS in small junctions where spatial variation of the rf-fields is neglected was very similar to the ordinary microwave-assisted tunneling (MAT) [6], [7] associated with the energy gap itself.

In order to verify the suggestion of Longacre and Shapiro we have made a careful series of measure-

(*) Permanent address, Department of Electrical Engineering, University of Rochester, Rochester 14627, New York. ments of the rf-power dependence of the SGS both for thin-film tunnel junctions (Sn-SnO-Sn) and for point-contact junctions (Nb-Nb). Figure 1 shows the $I-V$ characteristic of a typical point-contact junction (trace $a$ ) and also the corresponding derivative, $\mathrm{d} V / \mathrm{d} I$, which more clearly exhibits the structure (trace $b$ ) with no applied rf. The effect of applied $4 \mathrm{~mm}$ radiation $(66 \mathrm{GHz})$ on the junction of figure 1 is illustrated in figure 2 which shows a selection of derivatice traces as functions of bias voltage each trace corresponding to a different level of incoming rfpower. The rf-response demonstrated by this figure is representative for all the junctions tested - both thin-film tunnel junctions and point-contact junctions - in that they all behaved in full agreement with the following results obtained from figure 2 .

Considering, first, the energy gap region we find that the structure at $V=2 \Delta / \mathrm{e}$ decreases as the power increases and that it is replicated on either side of the gap voltage spaced by $\sim 270 \mu \mathrm{V}$ corresponding to a separation $h v / \mathrm{e}$ at $66 \mathrm{GHz}$. This is recognized as MAT [6] in the limit of high frequencies (this limit is defined by the photon energy, $h v / \mathrm{e}$, being greater than the width of the gap structure). Secondly, we find that the peak at half the gap voltage also decreases with increasing power and at a faster rate than did the energy gap structure. Here, replicas (to be named satellites) grow up on either side spaced by $\sim 135 \mu \mathrm{V}$, i. e., by $h v / 2$ e. Thirdly, the peak at one third of the gap voltage is seen to decrease still faster with increasing power and at the higher power levels it is seen 


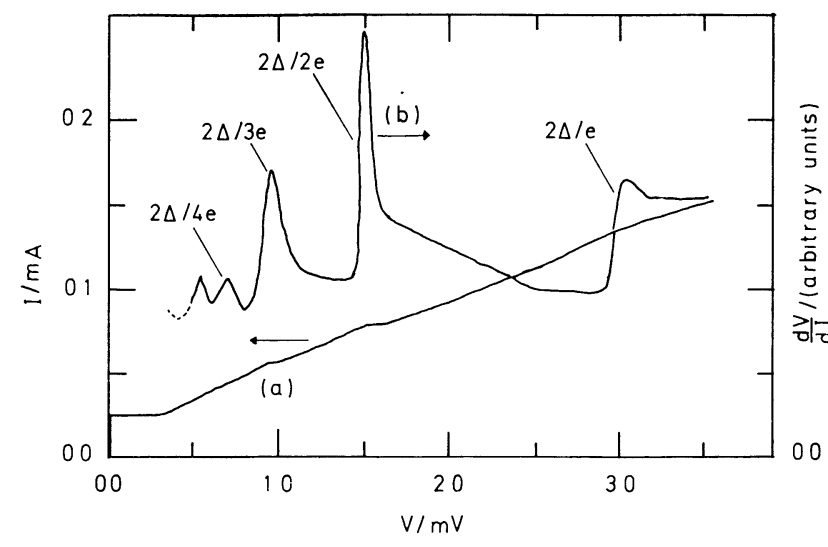

Fig. 1. - Current-voltage characteristic for Nb-Nb pointcontact junction showing subharmonic gap structure (trace $a$ ). The corresponding derivative, $\mathrm{d} V / \mathrm{d} I$, is also shown (trace $b$ ). No applied radiation. Normal state resistance $27 \Omega$, temperature $4.2 \mathrm{~K}$.

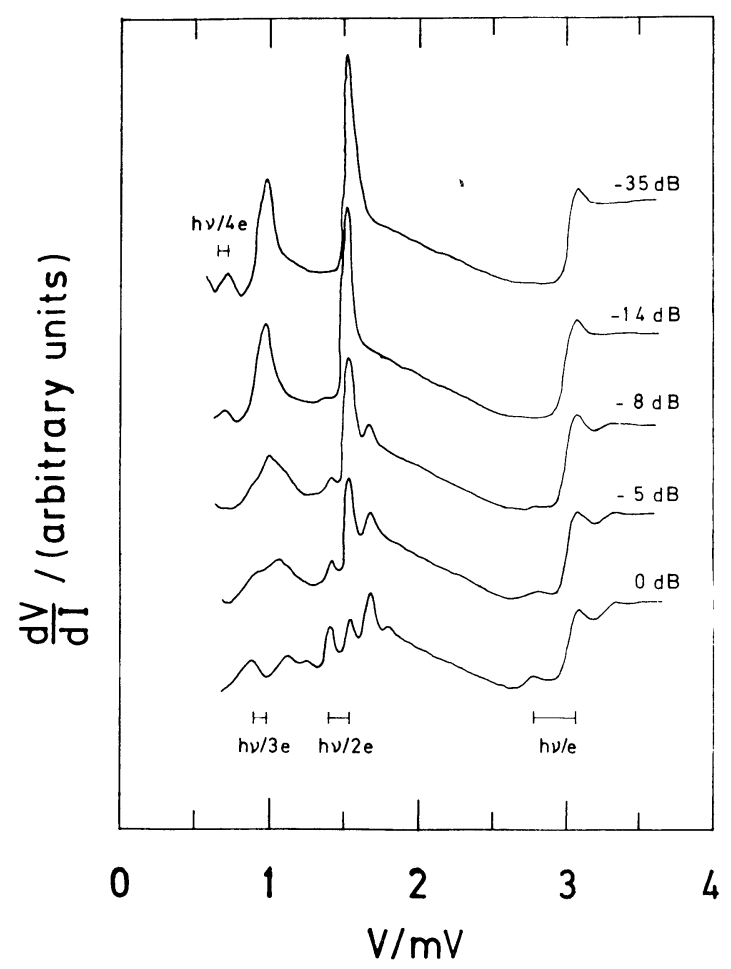

FIG. 2. - Selected derivative traces for the junction of figure 1 showing the effect of applied rf. The individual traces have been displaced vertically for clarity, and the numbers associated with each trace give the incoming rf-power in $\mathrm{dB}$ (the readings of a calibrated attenuator). The bar show the magnitude of $h v / m e$ for $m=1,2,3$, and 4 respectively corresponding to a frequency

$$
v=66 \mathrm{GHz} \text {. }
$$

to split into two peaks spaced by $2 h v / 3$ e corresponding to a satellite separation of $h v / 3$ e. Finally, also the peak at one fourth of the gap voltage decreases. No satellites are, however, resolved in this case.

By analogy to the well understood MAT these qualitative observations lead to the "ansatz » that the structure at the voltage $V=(2 \Delta \pm n h v) / m e$ vary with rf-power as squared Bessel functions, $J_{n}^{2}(m \alpha)$, of order $n$ and argument $m \alpha$ where the parameter $\alpha=\mathrm{e} V_{\mathrm{rf}} / h v$ is the ratio between the induced microwave voltage, $V_{\mathrm{rf}}$, across the junction and the photon energy (in electronvolts). This includes the ordinary MAT as a special case $(m=1)$.

In order to test this ansatz the amplitudes of the structures have been measured. Figures $3 a$ and $3 b$ show the normalized amplitudes of the $n=0$ and $n=1$ structures respectively for $m$ values as indicated plotted as functions of the incoming rf-power. Also shown are the corresponding squared Bessel functions (solid lines) as functions of the parameter $\alpha^{2}$. Comparison between the experimental results and the Bessel functions can be made by assuming proportionality between $\alpha^{2}$ and rf-power, and the scaling factor between the two has been fixed for all the data by fitting $J_{0}^{2}(2 \alpha)$ to the point at the arrow.

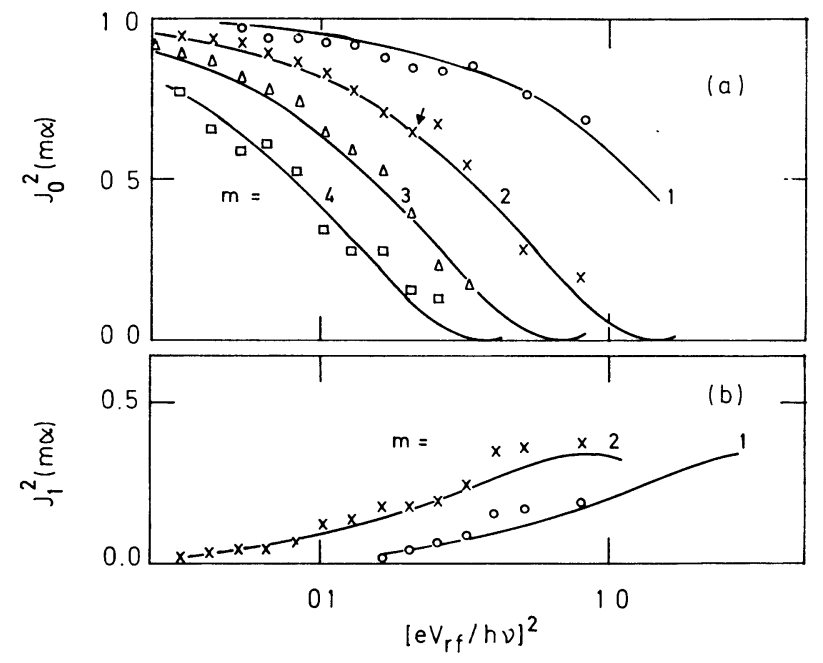

Fig. 3. - The measured amplitudes of the subharmonic gap structures $(a)$ and of the satellites $(b)$. The results are for any given $m$ normalized to the amplitude of the $n=0$ structure in the limit of zero rf-power and are plotted as functions of the incoming power.

(a) Shows the $n=0$ structures for $m=1$ (circles), $m=2$ (crosses), $m=3$ (triangles) and $n=4$ (squares).

(b) Shows the $n=1$ structures for $m=1$ and 2 with symbols as above.

Also shown are the corresponding squared Bessel functions, $\tilde{J}_{n}^{2}(m \alpha)$ (solid lines).

Good agreement between the experimental results presented here and $J_{n}^{2}(m \alpha)$ has been obtained in this way indicating that the ansatz is justified. A more detailed account for the experimental results obtained on a large number of junctions will be published elsewhere [8]. Here, we note only that all results obtained so far are in full agreement with the following concluding statement:

The amplitude of the structure observed at $V=2 \Delta / m$ e where $m=1,2,3, \ldots$, vary with rfpower as $J_{0}^{2}(m \alpha)$ where $\alpha$ is related to the induced microwave voltage, $V_{\text {rf }}$, by $\alpha=\mathrm{e} V_{\text {rf }} / h v$ and where $v$ 
is the applied frequency. In addition satellite structures are observed at $V=(2 \Delta \pm n h v) / m$ e with $n=1,2,3, \ldots$, and the amplitude of these satellites vary with power as $J_{n}^{2}(m \alpha)$.

Hasselberg, Levinsen and Samuelsen [9] have derived the power dependence predicted by multiparticle tunneling and nonlinear self-coupling and have found that they both agree with our experimental results. Hence, a distinction between these theories cannot be made on this basis.

Acknowledgments. - We gladly acknowledge the interest and encouragement of Professor K. Saermark at all stages of this work.

\section{References}

[1] Rowell, J. M. and Feldman, W. L., Phys. Rev. 172 (1968) 393.

[2] SchriefFer, J. R. and Wilkins, J. W., Phys. Rev. Lett. 10 (1963) 17.

[3] Werthamer, N. R., Phys. Rev. 147 (1966) 255.

[4] Giaever, I. and Zeller, H. R., Phys. Rev. B 1 (1970) 4278.

[5] Longacre, A., jr. and Shapiro, S., Bull. Am. Phys. Soc. II 16 (1971) 399.
[6] Tien, P. K. and Gordon, J. P., Phys. Rev. 129 (1963) 647.

[7] Hamilton, C. A. and Shapiro, S., Phys. Rev. B 2 (1970) 4494.

[8] Hoffmann Soerensen, O., Kofoed, B., Pedersen, N. F. and Shapiro, S. Phys. Rev. (to be published).

[9] Hasselberg, L. E., Levinsen, M. T. and Samuelsen, M. R. Revue Phys. Appl. 9 (1974) 157. 